\title{
Neurooncology: current findings and challenges for the future
}

\author{
Silvia Hofer
}

Received: 28 May 2013 / Accepted: 29 May 2013 / Published online: 22 June 2013

(C) Springer-Verlag Wien 2013

Primary central nervous system (CNS) tumors comprise approximately of 120 histological subtypes. Thus, it seems reasonable to concentrate primarily on some common entities. Most primary CNS tumors of adults are of glial origin and if they belong to WHO grade II-IV, they diffusely infiltrate the normal brain ab initio, i.e., pretherapeutically. With current standard treatment they cannot be cured. And, here lies an immense challenge for the neurooncology community.

Gliomas are defined by histomorphological criteria, described in the fourth edition of the WHO classification, published 2007 [1]. Histomorphology itself is able to precisely describe histological variants of CNS tumors and add new entities as soon as they are recognized by an international Working Group of 25 neuropathologists around the world. However, biological behavior of a certain tumor is only imperfectly predictable by histomorphological criteria alone.

There is now growing interest in the identification and validation of diagnostic, prognostic, and predictive molecular markers to divide brain tumors into biologically distinct categories, in addition to histomorphological criteria. Specific genetic changes (e.g. IDH1 mutation, LOH $1 \mathrm{p} / 19 \mathrm{q}$, and MGMT methylation status) segregate prognostic glioma subtypes and some have already proven useful in decision making to predict systemic treatment. Apart from tissue and blood derived biomarkers, "old" established and proven clinical prognostic factors, such as age, Karnofsky performance status, extent of resection, among others, are easily available without any additional costs and are still the part of a clinicians armamentarium to guide treatment choices.

\section{S. Hofer, MD $(\bowtie)$}

Department of Oncology, University Hospital Zürich, Rämistrasse 100, 8091 Zürich, Switzerland

e-mail: silvia.hofer@usz.ch
Two biomarkers that are newly integrated into clinical practice deserve a more detailed description.

For anaplastic WHO grade III oligodendrogliomas, the loss of $1 \mathrm{p} / 19 \mathrm{q}$ (LOH $1 \mathrm{p} / 19 \mathrm{q})$ due to an unbalanced translocation improves both survival and the response to therapy, and is thus, both a prognostic and a predictive marker. The most recent long-term follow-up from two large randomized Phase III trials [2, 3] call for a routine testing of this marker with PCR or FISH technique in WHO grade III gliomas. A marked overall survival benefit was achieved from the addition of chemotherapy to radiation confined to patients with anaplastic oligodendroglial tumors with, versus without, $1 \mathrm{p} / 19 \mathrm{q}$ co-deletion.

Promoter methylation of the MGMT gene causes epigenetic silencing of the methyltransferase, which loses its gene repair activity if inactivated. Evidence is now available for the growing population of elderly glioblastoma (GBM) patients, unfit for postoperative concomitant radiation- and chemotherapy to guide treatment decision upon MGMT methylation status. A methylated MGMT promoter (i.e. silenced gene) is a powerful predictive biomarker for the benefit of postoperative chemotherapy with temozolomide alone. For unmethylated tumors however, radiation therapy alone is considered the standard in this patient population. This has been clearly shown by two randomized trials with patients older than 65 and 60 years respectively $[4,5]$. In the absence of alternative therapeutic strategies, younger and fit patients with a GBM ( $<70$ years) should be offered postoperative concurrent chemo-irradiation without necessarily testing for the MGMT status.

Determination of the methylation status of the MGMT promoter, however, is technically demanding and requires reproducible and validated test procedures. Various techniques are available and a generally accepted consensus is still pending. Yet, a methylation-specific 
polymerase chain reaction (MSP) seems appropriate for clinical routine testing with low sample numbers.

Mutations of still another marker, namely of the isocitrate dehydrogenase (IDH) genes have been identified as an early event in gliomagenesis. These mutations are a typical feature of low-grade glioma, secondary (transformed) higher grade glioma and in a small cohort of GBM, and are associated with a more favorable prognosis. IDH mutations result in an oncometabolite, called 2-hydroxyglutarate (2HG). Subsequent accumulation of this metabolite in the tumor tissue interferes with the epigenetic machinery and induces a CpG island methylator phenotype. Recent advances have shown that magnetic resonance spectroscopy is able to detect $2 \mathrm{HG}$ in a non-invasive manner. Ongoing research is aimed at targeting this enzyme for therapeutic purposes.

A biomarker-led understanding of anti-angiogenic agents is highly desirable, but no such marker has been reliably identified so far. Vascular endothelial growth factor (VEGF), a key regulator of angiogenesis, is highly expressed in GBM. Bevacizumab, a monoclonal antibody that binds to VEGF is able to block edema formation; the true value on tumor growth however remains unclear, although the drug seems to be of benefit in selected patients with recurrent glioma.

So far no molecular alteration has been validated to render a GBM susceptible to a molecularly targeted therapy in a prospective study. Potentially useful molecular markers (e.g. c-met) remain to be validated in prospective randomized trials including multivariate analyses to guide clinicians in decision making. As treatment paradigms evolve toward a more personalized approach, it will become more imperative to define the patient population most likely to benefit from a treatment. Thus, health costs can be saved and side effects spared for those patients not expected to draw any benefit from the treatment.

Although very rare in adults, medulloblastomas are mentioned here, because they are a beautiful example of a systematic molecular profiling that allowed the identification of at least four distinct molecularly defined subgroups. They correspond to different clinical features and outcome. Insights into disease pathogenesis permit novel targeted treatments (e.g. sonic hedgehog pathway and corresponding inhibitors), currently being under clinical evaluation [6].

In diseases with extremely limited lifespan, a special focus has to be directed to quality of life (QoL) issues and not solely to length of progression free and overall survival. Several patient-related, treatment-related (e.g. neurotoxicity), and sociocultural factors influence QoL. The EORTC (European Organization for Research and Treatment of Cancer) has-some 20 years ago-set out to introduce and validate the EORTC Core Quality of Life questionnaire (EORTC QLQ-C30) which has been translated into 81 languages and is designed to measure cancer patients' physical, psychological, and social func- tions. For brain tumor patients, a brain cancer module (EORTC QLQ-BN20) exists, which is widely used to assess health-related QoL and symptoms, with special concerns regarding cognitive dysfunction and personality changes [7]. Consequently, the clinical benefit of any new treatment should be weighed against treatment side-effects that adversely influence patients' QoL. Well-designed trials take this requirement into consideration and one might expect a positive QoL endpoint to have an influence on the approval of a novel drug.

Along this line the EORTC is currently validating screening tools to assess the functional status and independence of a patient pre-therapeutically, an endeavor addressing particularly the population of the elderly.

Better tumor characterization together with a thorough assessment of the patient's condition will allow a more precise identification of patients most susceptible to a specific treatment.

\section{Conflict of interest}

The author declares that there is no conflict of interest.

\section{References}

1. Louis D, Ohgaki H, Wiestler O, et al. The 2007 WHO classification of tumors of the central nervous system. Acta Neuropathologica. 2007;114:97-109

2. Cairncross G, Wang M, Shaw E. Phase III trial of chemoradiotherapy for anaplastic oligodendroglioma: long-term results of RTOG 9402. J Clin Oncol. 2013;20, 31(3):337-43

3. van den Bent MJ,BAA van, Taphoorn MJ, et al. Adjuvant procarbazine, lomustine, and vincristine chemotherapy in newly diagnosed anaplastic oligodendroglioma: long-term follow-up of EORTC brain tumor group study 26951. J Clin Oncol. 2013;20, 31(3):344-50

4. Wick W, Platten M, Meisner C, et al. NOA-08 Study Group of Neuro-oncology Working Group (NOA) of German Cancer SocietyNOA 08. Temozolomide chemotherapy alone versus radiotherapy alone for malignant astrocytoma in the elderly: the NOA-08 randomised, phase 3 trial. Lancet Oncol. 2012;13(7):707-15

5. Malmström A, Grønberg BH, Marosi C, et al. Nordic Clinical Brain Tumour Study Group (NCBTSG). Temozolomide versus standard 6-week radiotherapy versus hypofractionated radiotherapy in patients older than 60 years with glioblastoma: the Nordic randomised, phase 3 trial. Lancet Oncol. 2012;13(9):916-26

6. Northcott PA, Jones DT, Kool M, Robinson GW, Gilbertson RJ, Cho YJ, Pomeroy SL, Korshunov A, Lichter P, Taylor MD, Pfister SM. Medulloblastomics: the end of the beginning. Nat Rev Cancer. 2012;12(12):818-34

7. Taphoorn MJ, Stupp R, Coens C, Osoba D, Kortmann R, van den Bent MJ, Mason W, Mirimanoff RO, Baumert BG, Eisenhauer E, Forsyth P, Bottomley A,European Organisation for Research and Treatment of Cancer Brain Tumour Group, EORTC Radiotherapy Group, National Cancer Institute of Canada Clinical Trials Group. Health-related quality of life in patients with glioblastoma: a randomized controlled trial. Lancet Oncol. 2005;6(12):937-44 